\title{
Navigating individual and collective interests in medical ethics
}

In medical ethics, we are often concerned with questions that pertain predominantly to the treatment of a particular individual. However, in a number of cases it is crucial to broaden the scope of our moral inquiry beyond consideration of the individual alone, since the interests of the individual can come into conflict with the interests of the wider community.

How should we resolve such conflicts between the interests of the individual and the collective? Most readers of this journal will likely be familiar with the moral theory 'classical utilitarianism', which enjoins us to bring about the greatest happiness for the greatest number. This theory offers straightforward guidance in such conflicts, since it will typically be the case that the interests of the many will outweigh the interests of the individual in the utilitarian's moral calculus. However, the simplicity of this approach is typically understood to be an inadequacy of the theory rather than an appropriate solution to these conflicts; contrary to the implications of classic utilitarianism, we would not believe it permissible to kill one innocent person in order to harvest life-saving organs for five others.

Of course, adherents of utilitarianism have responded to this sort of problem by refining the theory in various ways. However, one may invoke different kinds of moral apparatus in order to navigate conflicts between the individual and the collective. In this issue, a number of papers engage with moral concepts that are central to different approaches to navigating these conflicts in practical debates.

\section{RIGHTS AND INFECTIOUS DISEASE CONTROL}

While it may clearly be in the interests of the collective to restrict the transmission of a dangerous infectious pathogen, methods of achieving this aim can be contrary to the interests of the particular individuals targeted by the intervention. Consider quarantine measures; in quarantine, individuals who are merely suspected of carrying an infectious pathogen have their freedom of movement and association severely restricted.

One might seek to invoke the concept of a right in an attempt to prevent a utilitarian justification of restrictive measures in infectious disease control. For instance, one might claim that quarantine violates the quarantined individual's freedom of movement and association; other measures, such as forced medical treatment and vaccination might plausibly violate other rights, such as a right to bodily integrity.

Libertarians take such rights particularly seriously, and we might thus expect that libertarians would be critical of non-consensual infectious disease control measures that infringe upon these rights. However, in this issue, Jason Brennan counters this assumption by arguing that libertarians should, in fact, endorse mandatory vaccination programme (see pp. 37-43). Crucially, his argument is not that individual rights against mandatory vaccination can simply be trumped by the overwhelming interests of the collective here. Neither can a Millian harm principle be invoked to justify overriding rights against mandatory vaccination (although Brennan suggests that this principle might more plausibly be invoked in defence of quarantine measures, which target individuals who represent a clear and present danger to others). Rather, Brennan grounds his libertarian argument for mandatory vaccination in the claim that libertarians should endorse his 'clean hands principle', according to which there is a (sometimes enforceable) moral obligation not to participate in collectively harmful activities, including exposing others to unacceptable risk. Insofar as those who refuse vaccines collectively impose unacceptable risk on others, the libertarian can plausibly justify mandatory coercive vaccination programme, without betraying their core principles.

Brennan's argument is a novel libertarian approach to vaccine policy, with a somewhat surprising result. Yet, some libertarians may have concerns about the potential scope of the argument's implications. Much here depends on one's interpretation of unacceptable risk, and of the kinds of right infringement that unacceptable risk imposition can justify. For Brennan, risk is only acceptable if 'it is part of an equitable social system of risk-taking that work to her advantage'. The question this raises is whether the libertarian must allow that any right can be overridden, if doing so is necessary to prevent the collective imposition of an unacceptable risk per se, or whether there are certain rights that can only be justifiably overridden once the risk imposition in question reaches a certain degree of unacceptability. For instance, suppose that a group is collectively exposing the community to a very small risk of an only somewhat burdensome disease, but without any benefit to the community. Even this small risk could qualify as unacceptable on Brennan's terms; however, even if it seems plausible that the libertarian could deem it permissible to override some rights, such as freedom of movement and association, to prevent the imposition of this small risk, it is far from clear that this risk could justify overriding arguably more robust rights, such as the right to bodily integrity and the right to life, even if such violations were necessary for preventing the collective risk imposition.

\section{RESPONSIBILITY AND JUST RESOURCE ALLOCATION}

The navigation of conflicts between individual and collective interests in other contexts may call for justice-based considerations other than rights. Consider the allocation of scarce medical resources. Individuals have an obvious interest in having access to resources that are required for their medical treatment. At the collective level though, it is not possible to act in accordance with every such individual's interests; there are simply not enough resources to go around. Moreover, the community has a collective interest in how the state allocates such resources. Accordingly, an individual's interest in accessing a scarce medical resource must be weighed against the competing needs of other individuals, and also the collective's interest in how its resources are allocated.

One might adopt a broadly utilitarian approach to managing this conflict by advocating that the state should seek to allocate its resources in a manner that will simply maximise Quality-of-Life-Adjusted-Life-Years across the population. However, objections to a utilitarian conception of justice have given rise to alternative approaches that invoke considerations of desert and fairness in priority-setting. One frequently-invoked consideration is that priority setting for scarce health resources should in some way take into account the individual's responsibility for their health need - call this the 'responsibility view'. 
Phoebe Friesen critiques the responsibility view in this issue (see pp. 53-58). As Friesen notes, one moral argument for the responsibility view claims that individuals who are responsible for their health needs deserve different forms of consideration in the allocation of scarce resources from those who are not responsible, because the former (but not the latter) have failed to fulfil an important set of obligations. She also notes that the responsibility view has been supported by consequentialist considerations, including the claim that holding people responsible for their illness will lead to positive health outcomes.

Friesen objects to such consequentialist justifications of the responsibility view in the second half of her article, while she considers the argument from desert in the first half. Here, she notes that the cases most frequently considered in the literature on the responsibility view concern socially unacceptable behaviour. However, there are also a number of cases left largely unconsidered in the literature, in which individuals seem to be responsible for their health need by virtue of engaging in a socially acceptable behaviour. In light of this asymmetry in consideration, she suggests that the responsibility view may plausibly have partly been grounded by biases against stigmatised behaviours. Accordingly, Friesen challenges proponents of the responsibility view to either expand the scope of the view to incorporate these unconsidered cases, or to provide an explanation for the focus on stigmatised behaviours within the considered cases.

Although he is broadly sympathetic to Friesen's arguments about bias in assessments of responsibility, Julian Savulescu takes up the second horn of this challenge in his commentary (see pp. 59-61). Here, he suggests an explanation of the asymmetry by claiming that the considered cases involve 'unreasonable risks' while the unconsidered cases involve 'reasonable risks'. However, he concedes that establishing that a risk is unreasonable does not entail that a person is responsible for taking that risk; the individual may yet lack sufficient control over their behaviour. Accordingly, Savulescu agrees with Friesen that we have good reasons not to take retrospective responsibility for past behaviour into account in priority-setting. However, he contends that it may be easier to establish prospective responsibility for future behaviour, and outlines a way in which priority setting might appropriately take such responsibility into account.

While there is a great deal of rich discussion in Friesen and Savulescu's exchange regarding the role of responsibility in priority setting, it is notable that neither question the fundamental moral principle underlying the responsibility view, namely the principle that just resource allocation requires prioritising in accordance with desert of the sort that can be influenced by one's responsibility. Instead, both Friesen and Savulescu criticise the (retrospective) responsibility view by raising concerns about assessing individual responsibility. However, by questioning the above moral principle, it may be possible to criticise the responsibility view without relying on the premise that retrospective responsibility for an individual's current health need cannot typically be established. Notably though, Savulescu's proposal of taking prospective responsibility into account would also be vulnerable to this line of criticism. Nonetheless, highlighting the significance of this principle is important in clarifying our understanding of the role that the concept of responsibility should play in navigating this particular conflict between individual and collective interests.

\section{RESEARCH ETHICS IN DISASTERS}

Finally, a revisionary approach to navigating these conflicts is to challenge what may be a dogmatic assumption that such a conflict actually obtains in a particular context. This is a strategy that Phillipe Calain adopts in his wide-ranging Feature Article examining research ethics in the context of clinical trials carried out during the Ebola epidemic (see pp. 3-8).

On one prominent understanding, medical research is distinguished from medical therapy on the basis that the primary aim of the former is to benefit the collective (by developing generalizable scientific knowledge), rather than an individual patient. On this approach, a significant role for research ethics is to outline appropriate safeguards (such as the requirement for clinical equipoise) that will sufficiently protect the interests of individual research participants in the pursuit of the collective benefits of scientific knowledge.

Despite the theoretical attraction of this neat separation between the competing individual-focused aims of therapy and collective-focused aims of research, Calain argues that this conventional understanding is ill-equipped to deal with the questions raised by clinical trials in epidemic emergencies, such as the Ebola crisis. Lessons from this context suggest that participants in such trials are motivated to participate by a hope of therapeutic benefit, rather than from altruistic motives of contributing to scientific knowledge; moreover, clinicians involved in such trials may be less willing to abandon their therapeutic role (in the way that some trial designs demand) than the conventional view of research ethics implicitly assumes. Furthermore, he suggests that the possibility of achieving clinical equipoise, a frequently-invoked criterion of ethical research, is jeopardised in fatal epidemics by the subjectivity and unverifiability of the concept in such circumstances. Accordingly, Calain argues that by using adaptive trial designs that forged a middle way between clinical trials and compassionate use, it was possible to reconcile the putative conflicts between the individual and the collective in the Ebola trials.

Calain concludes by suggesting that this represents an important stage in the evolution of our understanding of therapeutic research, with consequences that go beyond 'disaster ethics', to the use of experimental interventions more broadly. This is a point that Bridget Haire takes up in her commentary (see pp. 9-10). In particular, she suggests that the 'collective vs individual' dichotomy in research ethics is often too simplistic, since the former concept admits of many different levels. On some of these conceptions, the interests of the collective may not be in conflict with the individual; moreover, in some cases, these particular conceptions of the collective should take ethical priority over others. Similarly, Annette Rid concurs with Calain's broad strategy of seeking to reconcile the apparent conflict between the individual and the collective in the conventional view of research ethics (see pp. 11-12). However, her diagnosis of the malaise in the conventional view differs from Calain's. The issue for Rid is not so much that the conventional view prioritises the collective over the individual, but rather that the conventional view lacks an account of investigators' positive obligations to promote participants' individual interests.

There is an important lesson for medical ethics quite generally in this exchange of articles. In some cases, we may become so concerned with the plausibility of using particular kinds of moral apparatus to navigate a conflict between collective and individual interests, that we may be blind to ways in which compromise may be achieved, and of practical strategies that we might employ to allow us to avoid this rocky theoretical terrain.

Funding JP is funded by the Wellcome Trust. Competing interests None declared.

Provenance and peer review Commissioned; internally peer reviewed.

(c) Article author(s) (or their employer(s) unless otherwise stated in the text of the article) 2018. All rights reserved. No commercial use is permitted unless otherwise expressly granted. 